\title{
Sodium valproate induced hyperammonaemia in an elderly patient with affective disorder
}

\author{
Kumanan Thirunavukarasu and Jayaprakash Russell Ravan
}

\section{Summary}

A 60 year old patient with bipolar affective disorder presented with clinical features suggestive of encephalopathy two weeks after commencing treatment with sodium valproate. He developed sudden onset alteration of sleep pattern, extreme agitation and hallucinations. He was disoriented in time, place, and person. Serum valproate level was within the therapeutic range. SGOT, SGPT, serum creatinine and echocardiogram were normal. Serum ammonia level which was $90 \mu \mathrm{g} / \mathrm{dl}$ (range: $25-80$ $\mu \mathrm{g} / \mathrm{dl}$ ) at the time of admission had risen to 180 $\mu \mathrm{g} / \mathrm{dl}$ on the 3rd day of hospital stay. Rapid clinical recovery from encephalopathy resulted a few days after withholding sodium valproate.

SL J Psychiatry 2011; 2 (1):31-32

\section{Introduction}

Sodium valproate is widely used as a mood stabiliser in the treatment of affective disorders. We report a case of an elderly patient with affective disorder on therapeutic doses of sodium valproate, who presented to the out patient department of a tertiary care hospital with clinical features suggestive of encephalopathy due to hyperammonaemia as a complication of sodium valproate treatment. Discontinuation of sodium valproate resulted in rapid clinical recovery. The clinical manifestations of encephalopathy in the elderly are atypical and may mimic worsening of psychosis or mania. Hence clinicians should be vigilant about the possibility of hyperammonaemia related encephalopathy in elderly patients on sodium valproate and a serum ammonia level may be indicated whenever such patients present with an altered sensorium.

\section{Case report}

A sixty-one year old male on treatment for recurrent depressive disorder developed mania with psychotic features while on treatment with antidepressants; hence the diagnosis was revised to bipolar affective disorder .The antidepressant was ceased and sodium valproate was introduced along with risperidone and diazepam. Initial investigations revealed no abnormalities in the total and differential blood count, serum glutamic oxaloacetic transaminase (SGOT), serum glutamic pyruvate transaminase (SGPT) and serum bilirubin. The serum creatinine level was normal at $1.0 \mathrm{mg} / \mathrm{dl}$ and the serum sodium valproate level, while on a dose of $1000 \mathrm{mg}$ per day was $102 \mathrm{ng} / \mathrm{ml}$. The patient became euthymic and regained a normal sleep pattern with treatment.

Two weeks later the patient presented with sudden onset alteration of his sleep pattern, extreme agitation and hallucinatory behaviour. Physical examination supported the diagnosis of encephalopathy. On mental state examination he was disoriented in time, place, and person. His serum valproate level was within the therapeutic range. His SGOT, SGPT, serum creatinine and echocardiogram results were within normal limits.
The patient's serum ammonia level which was $90 \mu \mathrm{g} / \mathrm{dl}$ (range: $25-80 \mu \mathrm{g} / \mathrm{dl}$ ) at the time of admission had risen to $180 \mu \mathrm{g} / \mathrm{dl}$ on the $3 \mathrm{rd}$ day of hospital stay and it was decided to withhold sodium valproate. This resulted in a rapid clinical recovery from encephalopathy in few days; however, biochemical normalcy was restored after three weeks.

\section{Discussion}

Sodium valproate is a widely used anti-convulsant and is also frequently used as a mood stabilizer in Bipolar Affective Disorder. It is known to have a high safety profile with occasional gastrointestinal side effects such as nausea, vomiting, anorexia, and hepatitis. Neurotoxicity has been documented in numerous case reports since 1973 and includes a spectrum of symptoms from drowsiness to stupor and coma. One of the earliest reports of sodium valproate associated hyperammonaemia appeared in 1980 in a case report by Caulter and Allen (1).

There are two mechanisms by which sodium valproate induced hyperammonaemia is believed to occur. The first mechanism is by inducing liver injury, which may be an idiosyncratic reaction to sodium valproate and usually occurs within three months of initiation of treatment. It is commoner in younger patients with underlying neurological illness and in those on multiple anticonvulsant therapy (2).

The second possible mechanism is associated with normal liver functions and without hepatic injury and was probably the mechanism by which this patient developed hyperammonaemia. It is related to the inhibition of two out of five mitochondrial enzymes in the urea cycle, namely carbamyl phosphate synthatase-1 and ornithine transcarbamylase $(3,4)$. The alteration of the sensorium appears to be related to the degree of hyperammonaemia. Patients presented with lethargy, stupor and coma and had ammonia levels of $>60 \mathrm{mmol} / 1$ (1). Blood ammonia levels did not correlate with the blood levels or dosage of sodium valproate (5). A study conducted in Japan suggested that age was not a risk factor for developing hyperammonaemia without liver injury.(6) 
Sodium valproate and one of its metabolites, propionate, are potent inhibitors of mitochondrial enzymes and inhibits carbamyl phosphate synthatase-1, the enzyme involved in the first step of removal of ammonia from the body $(3,7)$. This inhibition could be the probable mechanism by which this patient developed hyperammonaemia.

As the patient was an elderly male without any significant past history suggestive of hyperammonaemia and considering that the ammonia levels normalised after withdrawing the offending drug, ornithine transcarbamylase deficiency, the commonest X-linked inherited cause of hyperammonaemia, was unlikely.

Reports from the South Asian region describing sodium valproate induced hyperammonemia related encephalopathy in an elderly person with a primary psychiatric disorder are rare, in comparison to the abundant literature of hyperammonemia induced encephalopathy in children suffering from seizure disorder described in case reports, mostly from western countries.

This case report highlights several issues of potential clinical significance. It raises the question as to whether caution should be employed in the use of sodium valproate in elderly patients with bipolar affective disorder, especially in the use of products with faster absorption technology.

This report also raises the possibility that sodium valproate induced hyperammonemia may be an underdiagnosed entity as it be misinterpreted as a psychosis or worsening of mania leading to improper management and increased mortality.

It is suggested that clinicians should be aware of, and sensitive to, the possibility of sodium valproate induced hyperammonemia as a possible diagnosis when patients on the drug develops altered states of consciousness.

\section{Declaration of interest \\ None}

\section{Kumanan Thirunavukarasu,}

Tutor, Christian Medical College- Vellore, India and

Head \&Senior Lecturer, Department of Medicine, Faculty

of Medicine, University of Jaffna, Sri Lanka

Jayaprakash Russell Ravan,

Consultant Psychiatrist, Department of Psychosocial

Rehabilitation, Christian Medical College- Vellore- 632

002, India.

\section{Corresponding author}

Kumanan Thirunavukarasu, Department of Medicine,

Faculty of Medicine, University of Jaffna, Sri Lanka

E mail:mtkumanan@yahoo.com

\section{References}

1. Coulter DL, Wu H, Allen RJ. Valproic acid therapy in childhood epilepsy. JAMA 1980; 244: 785-8.

2. Raskind J, El-Chaar G. The role of carnitine supplementation during valproic acid therapy. The Annals of Pharmacotherapy 2000;34:630-638.

3. Coude FX, Rabier D,Cathelineau L,et al.Letter to the editer: Amechanism for valproate induced hyperammonaemia. Paediatric research 1981; 15:974975.

4. Rawat S,Borkowski W, Swick H. Valproic acid and secondary hyperammonaemia. Neurology 1981; 31:1173-1174.

5. Zaret BS,Beckner RR,Marini AM,wagle W,Passaralli C. Sodium valproate- induced hyperammonaemia without clinical hepatic dysfunction. Neurology 1982; 32(2):206-208.

6. Kondo T, Ishida M, Kaneko S, et al. Is 2-propyl-4phentenoic acid, a hepatotoxic metabolite of valproate, responsible for valproate induced hyperammonaemia? Epilepsy 1992;33(3):550-554.

7. Duarte J, Macias S,Coria F, Fernandez E, Calveria LE. Valproate induced coma: case report and literature review. The Annals of pharmacotherapy 1993; 27(5):582-583. 